\title{
The process of informed consent for urgent abdominal surgery
}

R Kay and A K Siriwardena Royal Infirmary of Edinburgh

\begin{abstract}
Objectives-To assess perceptions of the informed consent process in patients undergoing urgent abdominal surgery.

Design-A prospective observational study was carried out using structured questionnaire-based interviews. Patients who had undergone urgent abdominal surgery were interviewed in the postoperative period to ascertain their perceptions of the informed consent process. Replies were compared to responses obtained from a control group undergoing elective surgery, to identify factors common to the surgical process and those specific to urgent surgery. Patients'perceptions of received information were also compared to the information perceived to have been provided by the consent obtainers.

Setting - Gastrointestinal surgical service of a university teaching hospital.

Patients - Seventy-four consecutive patients undergoing urgent abdominal surgery and 80 control patients undergoing elective surgery.

Main measurements-Principal outcome measures were patients' perceptions of factors interfering with the ability to give informed consent, assessment of the quality of informed consent and the degree of discussion of the expected outcomes.

Results-Forty-nine of the seventy-four (66\%) patients undergoing urgent surgery perceived that pain did not affect their ability to give informed consent. Twenty-seven reported an adverse effect of analgesia on the ability to give informed consent. Only $22 \%$ of patients undergoing urgent surgery perceived that there had been any discussion of potential side effects and complications of surgery.

Conclusion-The majority of patients in this series with acute intra-abdominal surgical conditions perceive that they retain the ability to give informed consent for surgery. There is a need for improved discussion of therapeutic options and likely outcomes.

(Fournal of Medical Ethics 2001;27:157-161)
\end{abstract}

Keywords: Informed consent; urgent abdominal surgery

\section{Introduction}

Informed consent is the process whereby a mentally competent patient agrees to undergo a procedure after discussion of the indications, alternatives, potential side effects and complications. ${ }^{2}$ In patients undergoing urgent abdominal surgery this process may be compromised by the fact that the individual is in pain, or is under the effects of analgesia or by the need to intervene rapidly in situations where the precise diagnosis and likely outcome are unknown. In a previous study we demonstrated that the majority of a small cohort $(31 / 49$ [63\%]) of patients perceived that they retained the ability to give informed consent for urgent abdominal surgery despite being in pain. ${ }^{3}$

The nature of urgent surgery makes objective evaluation of the informed consent process difficult. Given the increasing array of therapeutic interventions available in urgent abdominal surgery and the increase in medicolegal activity relating to acute general surgery, however, the process of informed consent in this setting assumes increasing importance. The aim of the present study was to examine perceptions of the quality of informed consent in patients undergoing urgent surgery and to compare these to responses obtained from a control group undergoing elective surgery within the same institution, to identify factors common to the surgical process and those specific to urgent surgery.

Specific issues examined by the study include the effects of pain and analgesia on patients' perceptions of the informed consent interview. In addition, as the acute surgical service involves dealing with patients who may be unfamiliar with both hospital and staff, the study explores whether patients undergoing urgent surgery were less likely to know the grade of the clinician participating in the informed consent interview. Similarly, as urgent surgery may involve a greater proportion of junior staff, the study examined whether the operating surgeon was involved in the informed consent discussion.

The principal components of informed consent examined by the study were whether patients understood why an operation had been required and whether they had knowledge of the likely outcomes.

\section{Methods}

STUDY DESIGN

A prospective observational study was carried out using a structured questionnaire-based interview technique. Patients who had undergone urgent abdominal surgery were interviewed. A control group of patients undergoing elective abdominal surgery was also interviewed. In order to obtain a balanced perspective of the informed consent 
interview the consent obtainers were also interviewed. These individuals were interviewed away from the bedside and using a separate questionnaire.

\section{QUESTIONNAIRE}

Data were recorded in response to a structured questionnaire (appendix). The questionnaire incorporated a minimental state examination (MMSE) to allow recognition of patients who were cognitively impaired at the time of interview. ${ }^{4}$ This structured questionnaire had been validated in an earlier study. ${ }^{3}$ All questions were put to patients by one interviewer (RK) who had no involvement in the process of delivery of care. Whenever possible, interviews were conducted between one and four days postoperatively, at the earliest time at which it was considered that the patient was sufficiently comfortable for such an interview to take place. The interview was of approximately 20 minutes duration and was conducted with the individual's consent in all cases. Permission was obtained from all consultant surgeons to study patients admitted under their care. Surgeons agreed to remain unaware of the dates of commencement and completion of the study in order to reduce any influence of the study on standard practice.

Doctors obtaining informed consent were identified from the signature on the consent form and were interviewed in the postoperative period using a separate questionnaire (appendix).

\section{PATIENTS}

Patients over the age of 16 years admitted to the Royal Infirmary of Edinburgh with acute abdominal pain and undergoing urgent abdominal surgery were studied. For the purposes of this study urgent abdominal surgery was defined as a procedure performed for a suspected acute condition where operation could not be deferred and was generally (but not exclusively) performed within 48 hours of admission. A consecutive series of 94 patients undergoing urgent abdominal surgery were studied. Twenty individuals were excluded from the final analysis for the following reasons: seven died in the peri-operative period, five scored lower than 25 on minimental state examination, five were too unwell for interview, one could not speak English and had given consent via an interpreter and for two patients it was felt inappropriate to interview (one because the spouse had been fatally injured in the accident precipitating the patient's urgent abdominal surgery and the other because previously unexpected carcinomatosis had been found at operation). Seventy-four individuals thus constituted the study population. The types of operations undergone by patients having urgent surgery are shown in figure 1. Eighty patients undergoing elective surgery during the study period constituted the control group. Details of the urgent and elective groups are seen in table 1 . The groups were not age and sex matched but none of the differences between groups were significant (Mann Whitney U-test).

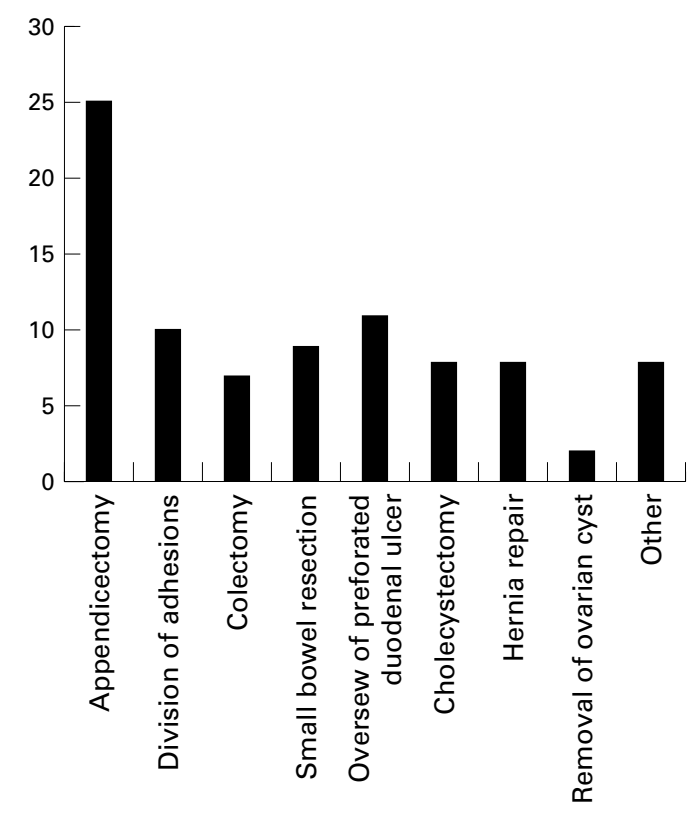

Figure 1 Types of urgent abdominal surgical procedures

\section{STATISTICAL ANALYSIS}

Quantitative data relating to details of patients in the urgent and elective surgery groups were compared by Mann Whitney U-test. Significance was accepted at the $P<0.05$ level. Direct comparison between the results obtained in the urgent and control groups is limited by the lack of truly comparable elective patient data. For example, the most frequently performed urgent abdominal procedure is appendicectomy and there is no such procedure as a truly elective appendicectomy. Where cautious comparisons have been made of variables to which both elective and urgent patients were exposed a $\chi$-squared analysis was employed. Statistical calculations were performed using the Instat ${ }^{\circledR}$ software package (GraphPad, Instat, California, USA).

Table 1 Details of patients admitted for urgent or elective abdominal surgery

\begin{tabular}{lll}
\hline Group & Urgent & Control \\
\hline Patient number & 94 & 82 \\
Exclusions & $20^{\star}$ & 2 \\
Study number & 74 & 80 \\
Male & 39 & 38 \\
$\begin{array}{l}\text { Mean age in years } \\
\text { Median delay from admission to }\end{array}$ & $51(16-94)$ & $54(23-85)$ \\
$\quad$ operation in days & $2(0-14)$ & $1(0-22)$ \\
$\quad \begin{array}{l}\text { Median delay from operation to } \\
\quad \text { interview in days }\end{array}$ & $1(0-18)$ \\
Mean MMSE & $28(15-30)$ & $29(18-30)$ \\
\end{tabular}

Values in parentheses are the range for each variable. 
ETHICAL APPROVAL

The study was approved by the student projects ethics committee of the University of Edinburgh Faculty of Medicine.

\section{Results}

PATIENTS' PERCEPTIONS OF FACTORS INTERFERING WITH THE ABILITY TO GIVE INFORMED CONSENT.

Sixty (81\%) patients undergoing urgent surgery were in pain at the time of consenting to undergo operation but only $25(34 \%)$ stated that pain interfered with their ability to give informed consent to surgery. No patient in the control groups reported pain interfering with the ability to give informed consent.

INFLUENCE OF ANALGESIA ON INFORMED CONSENT Of patients undergoing urgent surgery $52(70 \%)$ had received analgesia before signing consent and $14(27 \%)$ of this group reported that painkillers impaired the ability to give informed consent. Twelve patients $(16 \%)$ reported that both pain and the effects of analgesia impaired the ability to give informed consent. In the control group 17 patients $(21 \%)$ were taking regular analgesia at the time of giving consent but none reported any adverse effects of medication on the ability to give informed consent.

KNOWLEDGE OF GRADE OF DOCTOR OBTAINING

CONSENT.

Patients were asked if they knew the grade of the doctor obtaining consent. If specific grading was not known they were offered the option of stating whether the doctor was junior, middle grade, senior or of stating that they did not know the grade. Of patients undergoing urgent surgery 15 (21\%) correctly identified the grade of the doctor obtaining consent. This compared to $31(39 \%)$ patients in the control group $\left(\chi^{2}=12.3, \mathrm{p}<0.01\right)$.

COMPREHENSION OF INDICATIONS FOR, AND

POTENTIAL SIDE EFFECTS OF, SURGERY

Sixty-five $(88 \%)$ patients undergoing urgent surgery reported they understood why an operation had been necessary compared to 77 (96\%) undergoing elective surgery.

Of patients undergoing urgent surgery 16 (22\%) perceived they had received an explanation of the potential side effects and complications of surgery. Of patients undergoing elective surgery $40(50 \%)$ felt that they had received an explanation $\left(\chi^{2}=\right.$ $7.74, \mathrm{p}<0.01)$

DOCTORS' QUESTIONNAIRE

Consent forms were countersigned by preregistration house officers in all patients in this study. None were countersigned by the operating surgeon. In the group undergoing urgent abdominal surgery $42 / 74(57 \%)$ doctors stated they had checked whether the patient had received analgesia prior to obtaining informed consent.
Doctors obtaining consent were asked whether they had explained the indications for surgery. Of the group undergoing urgent surgery an explanation of the need for surgery had been provided in 62 $(84 \%)$. This compared to $63(79 \%)$ of the control group. In situations where indications were not explained by the consent obtainer, seven of 12 patients in the urgent surgery group had discussed the indications for surgery with a more senior doctor. In patients undergoing elective surgery, all 17 patients in whom the consent obtainer did not discuss the operation received information from a more senior doctor during that admission.

Doctors explained potential side effects and complications of surgery to $35 / 74$ (47\%) patients undergoing urgent surgery compared to $37 / 80$ $(46 \%)$ undergoing elective procedures. When side effects had not been discussed, a senior doctor had already discussed these in $20 / 39(51 \%)$ in the urgent surgery group and in all $43(100 \%)$ in the elective surgery group.

In order to control for any potential bias introduced by doctors becoming familiar with the content of the structured questionnaire, the patterns of responses for the first four weeks were compared to those obtained in the second fourweek period. There was no difference in numbers of doctors checking whether analgesia had been prescribed or in the numbers discussing indications and side effects ( $p=N S$; Mann Whitney U-test).

\section{Discussion}

This study examines informed consent in patients undergoing urgent abdominal surgery and compares the results to those obtained from a control group undergoing elective surgery. Structured interviews conducted in the postoperative period were used as this was thought to best represent the perceptions the patients would take with them from the inpatient episode.

When interpreting this study and the relevance of its findings the limitations imposed as a result of study design must be considered. First, conduct of the interviews in the post-operative period may have resulted in information given prior to surgery being forgotten by the patient. In this context Hekkenberg and colleagues have previously demonstrated that the elderly and individuals with lower levels of education are more likely to forget information given to them to permit informed consent. $^{5}$ Patients interviewed in the postoperative period may also have impaired recall of preoperative pain and the effects of pre-operative analgesia.

Would more representative results have been obtained if the interviews had been conducted prior to surgery? Pre-operative interview carries with it the risk of interference with the process of care ${ }^{6}$ For example, asking patients whether they had received information on the potential side effects and complications of surgery before they had undergone operation (and taking into consideration the findings of this study that the majority stated they received no information on these areas) may have 
caused unnecessary distress and have required further pre-operative interview by the doctors obtaining consent. Whilst this may have been beneficial for the patients in the present study, repeat interviews prompted by the interviews for this study would represent a distortion of the normal informed consent process. Thus, pre-operative interview was not employed. An exact record of the interview could also have been obtained by the use of video-recording techniques, but this would have produced a completely artificial "clinical" setting and recording was not selected. Postoperative interview, with the interview being conducted as early as possible in the postoperative period appeared a pragmatic compromise.

A second limitation of the study may have been introduced by the decision to interview the consent obtainers. Although it was argued that this would offer a balanced perspective of the informed consent interview it is also possible that the process of interview may have "primed" doctors and influenced their subsequent interviews. However, comparison of data from the first four weeks of the study compared to the second showed no evidence of such a trend. Finally, an inherent limitation in studies of urgent abdominal surgery is the lack of a truly representative control group.

\section{Previous study}

Within these limitations $81 \%$ of patients in our series undergoing urgent abdominal surgery were in pain at the time of giving consent to surgery. However, the majority $(66 \%)$ perceived that this did not affect their ability to give informed consent. This is consistent with the findings of our previous study. ${ }^{3}$ This agreement between our two studies supports the impression that at least some patients with acute abdominal pain perceive that they retain the ability to give informed consent and is interesting, given that the two studies were separated by a time period of over 12 months and involved two distinct cohorts of junior hospital doctors.

Although $52(70 \%)$ patients undergoing urgent surgery had received analgesia before signing consent only $14(27 \%)$ reported an adverse effect of analgesia on the ability to give informed consent. This is perhaps fortunate as in only $57 \%$ of cases did the consent obtainer ascertain whether or not the patient had received analgesia prior to the informed consent interview. However, data on patients' perceptions of the effects of analgesia may be unreliable because analgesia may impair memory.

It is of concern that only $16(22 \%)$ patients undergoing urgent surgery felt they had received an explanation of any potential side effects and complications of surgery. Similar deficiencies in explanation of the nature of urgent surgery were highlighted by Johnson and colleagues in a survey of patients' satisfaction with surgical services. ${ }^{7}$ Even in elective situations where written information is provided, patients may have impaired recall of the risks and nature of surgery. ${ }^{8}$ Thus the problems of providing sufficient information in urgent surgery where the precise findings and outcomes are unknown prior to intervention are considerable.

Taking into consideration the limitations imposed by study design the study reveals that there were appreciable differences in perceptions of informed consent between patients undergoing urgent and elective surgery. It is perhaps no surprise that patients undergoing elective surgery were better informed, having had the advantage of discussing their condition and planned treatment with a senior doctor prior to surgery. In contrast, the very nature of urgent surgery can engender a sense of loss of control from the patient's perspective. This degree of urgency is probably the principal determinant of the opportunity for adequate informed consent. The impression gained from the present study is that urgent surgery cannot be grouped together as a single entity. For example, in the clinical setting of penetrating abdominal trauma or acute conditions associated with major blood loss such as ruptured abdominal aortic aneurysm there is neither demand nor any opportunity for a detailed discussion of treatment options. In this situation it is clearly the doctor's duty to act in the best interests of the patient. ${ }^{2}$ In contrast, in the relatively common clinical urgency settings of acute appendicitis or acute cholecystitis treatment plans should be formulated with patient input. In acute appendicitis, there is evidence of near equal therapeutic efficacy from laparoscopic or open appendicectomy. ${ }^{9}$ It would seem appropriate at least to discuss these options with patients. Similarly, early or delayed laparoscopic cholecystectomy for acute cholecystitis may achieve the same final outcome but at the expense of a higher conversion and complication rate in the deferred surgery group. ${ }^{10}$ As with the decision to treat appendicitis by open or laparoscopic surgery it seems appropriate to involve patients in this decision making process.

Although the majority of patients undergoing both urgent and elective surgery stated they understood the need for surgery, there were significant differences in perceptions of the quality of discussion of the side effects and potential complications between urgent and elective groups. Previous studies have highlighted perceived dissatisfaction with this aspect of the quality of care of patients undergoing urgent surgery. ${ }^{3}$

\section{Ethical issues}

The quality of informed consent may be improved by increasing awareness of the ethical issues surrounding consent. ${ }^{112}$ On a more practical level the provision of information sheets addressing frequently raised issues relating to operations such as appendicectomy where the common range of outcomes can be reasonably covered may support the informed consent interview. An important caveat is that written information sheets do not replace the informed consent interview and that a tendency to provide excessive information must be avoided, with information being tailored to the issues raised by patients. ${ }^{13}$ 
In summary, this study has revealed that an appreciable proportion of patients undergoing surgery for acute abdominal conditions perceive that they retain the ability to participate in a discussion of informed consent. Informed consent for urgent surgery is perceived to be less comprehensive than for elective procedures. As the majority of patients in this study perceived no discussion of the potential side effects or complications of urgent surgery it could be argued that consent was not truly informed.

Procedural modifications such as ensuring that the surgeon carrying out the operation countersigns the consent form and alteration of the generic consent form to permit procedure-specific modifications may improve patient satisfaction in this important area of surgical activity.

\section{Appendix}

PATIENTS' QUESTIONNAIRE

1. Were you in pain at the time of your consent interview?

2. Did you want information about your operation?

3. Did pain restrict your ability to ask questions?

4. Had you received painkilling tablets or injections prior to your consent interview?

5. Did these restrict your ability to ask questions?

6. Did anything else restrict your ability to ask questions?

7. If yes, specify:

8. What was the grade of the doctor conducting the consent interview?

9. Did you understand why an operation was being planned?

10. If not, why was this?

11. Was there a discusion of the possible side effects and complications?

12. What possible side effects and complications were discussed?

a. Postoperative pain

b. Risk of wound infection

c. Predicted time in hospital

d. Predicted time off work

e. Impairment of driving

f. Need for medication after surgery

g. Any other specific side effects/complications

13. If there was no discussion of side effects, why do you think this was?

14. Who was the best source of information about your operation? (Doctor, nurse, family, friend, pamphlet, magazine, other)

15. Have you had an abdominal operation before?

16. With respect to the statement "I was able to give informed consent to operation" do you; 5=strongly agree, 4=agree, 3=uncertain, $2=$ disagree, $1=$ strongly disagree
DOCTORS' QUESTIONNAIRE

1. Who asked you to obtain consent?

2. Did you check whether the patient had received analgesia prior to the interview?

3. Did you assess the conscious level of the patient prior to interview?

4. Did you explain indications for the operation?

5. Had someone already done this? If so, who?

6. Did you explain potential side effects/ complications of the operation? If not, why not?

7. Had someone else already done this? If so, who?

8. Did you discuss alternative therapeutic options or types of operation?

9. Did anyone else discuss alternative therapeutic options or types of operation?

$R$ Kay $M B, C h B$, is a House Officer at the University Department of Surgery, Royal Infirmary of Edinburgh. A K Siriwardena MD, FRCS, is a Consultant Surgeon at the Department of Surgery, Manchester Royal Infirmary.

\section{References}

1 Medical Defence Union. Consent to treatment. Manchester: Medical Defence Union, 1996. General Medical Council. Seeking patients' consent: the
considerations. London: General Medical Council, 1998.

considerations. London: General Medical Council, 1998.
Vessey W, Siriwardena AK. Informed consent in patients with Vessey W, Siriwardena AK. Informed consent in patients with
acute abdominal pain. British fournal of Surgery 1998; 85:127880 .

4 Folstein MF, Folstein SE, McHugh PR. 'Mini-mental state'. A practical method for grading the cognitive state for clinicians. fournal of Psychiatric Research 1975;12:189-98.

5 Hekkenberg RJ, Irish JC, Rotstein LE, Brown DH, Gullane PJ. Informed consent in head and neck surgery: how much do patients actually remember? fournal of Otolaryngoly 1997;26: $155-9$

6 Myles PS, Fletcher HE, Cairo S, Madder H, McRae R, Cooper $\mathrm{J}$, et al. Randomized trial of informed consent and recruitment for clinical trials in the immediate pre-operative period. Anesthesiology 1999;91:969-78.

7 Johnson RC, Pye JK, Scriven MW, Billings PJ, Wood C, Crumplin MK. Patients' perception of surgical services in a district

.

Kroner S, W Gschwantler M. Patients' assessment and recall of surgical information after laparoscopic cholecystectomy. Digest of Surgery 1998;15:669-73.

9 Sauerland S, Lefering R, Holthausen U, Neugebauer EA. Laparoscopic vs conventional appendectomy-a meta-analysis of randomized controlled trials. Langenbeck's Archives of Surgery 1998;383:289-95.

10 Lo C-M, Liu C-L, Fan S-T, Lai ECS, Wong J. Prospective randomized study of early versus delayed laparoscopic cholecystectomy for acute cholecystitis. Annals of Surgery 1998;227: 461-7.

11 Angelos P, Da Rosa DA, Derossis AM, Kim B. Medical ethics curriculum for surgical residents: results of a pilot project. Surgery $1999 \cdot 126: 701-5$.

12 Nandi PL. Ethical aspects of clinical practice. Archives of Surgery 2000;135:22-5.

13 Newton-Howes PA, Bedford ND, Dobbs BR, Frizelle FA. Informed consent: what do patients want to know? New Informed consent. What do patients

14 Osuna E, Perez-Carceles MD, Perez-Moreno JA, Luna A. Informed consent. Evaluation of the information provided to patients before anaesthesia and surgery. Medicine and Law 1998;17:511-8 